\title{
Accurate spline solutions of the Dirac equation with parity-nonconserving potential
}

\author{
L.Labzowsky ${ }^{1,2}$ and A.Prozorov ${ }^{1}$ \\ 1 St Petersburg State University, 198504 Petrodvorets, St Petersburg Russia \\ 2 Petersburg Nuclear Physics Institute 188300 Gatchina, St Petersburg Russia
}

(October 31, 2018)

\begin{abstract}
The complete system of the B-spline solutions for the Dirac equation with the paritynonconserving (PNC) weak interaction effective potential is obtained. This system can be used for the accurate evaluation of the radiative corrections to the PNC amplitudes in the multicharged ions and neutral atoms. The use of the scaling procedure allows for the evaluation of the PNC matrix elements with relative accuracy $10^{-7}$.
\end{abstract}

PACS number(s): 31.30.Jv, 31.10.+z

\section{INTRODUCTION}

Consideration of the parity nonconservation (PNC) effects in atoms provides an important verification of the Standard Electroweak Model in the low energy sector. The most important information provides the neutral $C s$ atom, because the experimental and the theoretical accuracy is the best in this case. The analysis performed in [1] and [2] has indicated a deviation of the measured weak charge value $Q_{w}$ from that predicted by the Standard Model by 2.5 standard deviations $\sigma$. Later this value was diminishing and growing up again in a series of works [3]- [7]. It was understood that the radiative corrections to the PNC amplitude play an important role. This question was investigated in [8]- [12] where both electron self-energy (SE) and vacuum polarization (VP) corrections were evaluated by different methods. However, in these calculations $\alpha Z$ expansion for $\mathrm{SE}$ ( $\alpha$ is the fine-structure constant, $Z$ is the charge of the nucleus), Uehling approximation for VP or other approximations were employed. To our mind the direct accurate calculations of SE and VP without the use of any approximations are desirable. For this purpose we propose in this paper the accurate spline solutions of the Dirac equation with the weak-interaction PNC potential.

Another possible area of application of PNC spline solutions could be the spectra of the Highly-Charged Ions (HCI). Though no experimental results are available here up to now, the analysis of the PNC amplitude in the two-electron ions can also provide an important test of the Standard Model [13]- [19]. The calculation of correlation effects in two-electron ions is much easier than in neutral atoms, but QED corrections are more important.

The rigorous direct way to the calculation of SE and VP corrections to the PNC amplitude (see [20]) is rather cumbersome. In order to avoid the difficulties and receive the accurate results we propose to use another way: accurate solutions of the Dirac equation with the weak-interaction PNC potential. Having got the solutions of the Dirac equation with this potential we can obtain self-energy corrections, using the standard methods [21] - [23] based on the spline approximation [24], [25]. In this work we solve the first part of the problem: the obtaining of the complete basis set of accurate solutions of the Dirac equation with the weak PNC potential. The main difficulty is the extreme smallness of the weak interactions in the atomic scale. To overcome this difficulty we change the weak interaction potential by scaling it in such a way that it becomes significant but still small enough to apply the perturbation theory. Then we can obtain the necessary PNC matrix elements by simple rescaling. Actually our numerical procedure appear to be so accurate that the scaling will become necessary only in few cases, for the most singular operators and the enhancement of the weak interaction potential will not exceed 10 times.

\section{DIRAC EQUATION WITH PNC WEAK INTERACTION}

We employ atomic units $m_{e}=e=\hbar=1$ throughout the paper. Consider stationary Dirac equation with the weak-interaction PNC potential

$$
\left[c \vec{\alpha} \vec{p}+V(r)+\beta c^{2}+V_{w}(r)\right] \psi(\vec{r})=E \psi(\vec{r})
$$

where $\vec{\alpha}, \beta$ are Dirac matrices, $\vec{p}, E$ are the electron momentum and energy, $c \approx 137.03599976$ is the speed of light, $V(r)$ is the Coulomb potential of atomic nucleus (pointlike or extended) and $V_{w}$ is the weak-interaction PNC potential. 
This potential (see, for example, [26]) looks like

$$
\begin{gathered}
V_{w}=\gamma_{5} W(r)=\gamma_{5} \frac{G_{F}}{2 \sqrt{2}} Q_{w} \rho_{\text {nuc }}(r) \\
\gamma_{5}=-\left(\begin{array}{cc}
0 & I \\
I & 0
\end{array}\right) \quad \begin{array}{l}
Q_{w}=-N+Z\left(1-4 \sin ^{2} \theta_{w}\right), \\
\rho_{\text {nuc }}(r)=\rho_{0}[1+\exp [(r-c) / a]]^{-1}
\end{array}
\end{gathered}
$$

where $\theta_{w}$ is the Weinberg angle, $\sin ^{2} \theta_{w} \approx 0.2230$ [27], $N$ is the number of neutrons in the nucleus, $Z$ is the number of protons, $G_{F}$ is the Fermi constant and $\rho_{n u c}(r)$ is the Fermi distribution for nuclear charge density. The eigenfunctions of the Dirac equation with PNC potential have no definite parity, therefore we assume

$$
\psi(\vec{r})=\left(\begin{array}{c}
\varphi(\vec{r}) \\
\chi(\vec{r})
\end{array}\right)=\frac{1}{r}\left(\begin{array}{c}
g_{n j l}^{1}(r) \Omega_{j l M}(\vec{n})+i g_{n j l}^{2}(r) \Omega_{j \bar{l} M}(\vec{n}) \\
i f_{n j l}^{1}(r) \Omega_{j \bar{l} M}(\vec{n})+f_{n j l}^{2}(r) \Omega_{j l M}(\vec{n})
\end{array}\right)
$$

where $\varphi(\vec{r})$ and $\chi(\vec{r})$ are the upper and lower components of Dirac bispinor, $g_{n j l}^{1}(r), g_{n j l}^{2}(r), f_{n j l}^{1}(r)$ and $f_{n j l}^{2}(r)$ are the radial functions, $\Omega_{j l M}$ and $\Omega_{j \bar{l} M}$ are spherical spinors with opposite parity. The equations for the components $\varphi, \chi$ are

$$
\begin{aligned}
& \left(E-V(r)-c^{2}\right) \varphi(\vec{r})-c(\vec{\sigma} \vec{p}) \chi(\vec{r})-W(r) \chi(\vec{r})=0 \\
& \left(E-V(r)+c^{2}\right) \chi(\vec{r})-c(\vec{\sigma} \vec{p}) \varphi(\vec{r})-W(r) \varphi(\vec{r})=0
\end{aligned}
$$

Inserting (4) in (5),(6) and separating out the angular variables we get

$$
\left[\begin{array}{cccc}
V(r) & 0 & -c\left(\frac{d}{d r}-\frac{\kappa_{j l}}{r}\right) & W(r) \\
0 & V(r) & W(r) & c\left(\frac{d}{d r}+\frac{\kappa_{j l}}{r}\right) \\
c\left(\frac{d}{d r}+\frac{\kappa_{j l}}{r}\right) & W(r) & V(r)-2 c^{2} & 0 \\
W(r) & -c\left(\frac{d}{d r}-\frac{\kappa_{j l}}{r}\right) & 0 & V(r)-2 c^{2}
\end{array}\right]\left[\begin{array}{c}
g^{1} \\
g^{2} \\
f^{1} \\
f^{2}
\end{array}\right]=\varepsilon\left[\begin{array}{c}
g^{1} \\
g^{2} \\
f^{1} \\
f^{2}
\end{array}\right]
$$

where $\varepsilon=E-c^{2}$ and

$$
\kappa_{j l}=\left\{\begin{array}{ccc}
l & \text { if } & j=l-\frac{1}{2} \\
-(l+1) & \text { if } & j=l+\frac{1}{2}
\end{array}\right.
$$

This system of equations for the radial functions was first derived in [28]. However in [28] only one certain solution of Eq (7) was obtained. Our finite goal requires the knowledge of the complete system of the solutions of Eq (7). For this purpose we use the B-spline approach [24], [25].

\section{PARITY-NONCONSERVING B-SPLINE APPROACH}

Using the Galerkin method (see [29] and [24]) we express the system of equations (7) in terms of the least action principle. We use MIT bag-model boundary conditions [30], employed also in [24]. In case of PNC functions these conditions look like

$$
\left\{\begin{array} { l } 
{ g ^ { 1 } ( R ) = f ^ { 1 } ( R ) } \\
{ g ^ { 2 } ( R ) = f ^ { 2 } ( R ) }
\end{array} \quad \left\{\begin{array}{l}
g^{1}(0)=0 \\
g^{2}(0)=0 .
\end{array}\right.\right.
$$

Here $R$ is the size of a spherical box, where an atom or an ion is enclosed. We choose action functional $S$ so that condition $\delta S=0$ leads to Eqs (7) and (9). Then we present the functions $g^{1}, g^{2}, f^{1}$ and $f^{2}$ in the form of the linear combination of B-splines. The condition $\delta S=0$ reduces to the system of $4 n \times 4 n$ symmetric generalized eigenvalue equations. For constructing the B-spline system we use the grid $r_{i}=\rho_{i}^{4} / Z, \quad i=0, \ldots, N_{i n t}$, that was described 
in [25]. In our calculations we use the number of the grid intervals $N_{i n t}=200$, the order of B-splines $k=9$ and the parameter, which defines the density of knots near zero (see [25]), $h_{\text {set }}=0.0167$.

In this paper we investigate the hydrogenlike ion ${ }^{238} U^{91+}$. The box size was taken to be $R \approx 1.0$ a.u. The parameters of the Fermi distribution are $c=7.136 \mathrm{fm}$ and $a=2.3 / 4 \ln 3$ [31]. As a first test of the accuracy of our B-spline approach, we set $V(r)=-Z / r, N_{w}=0$ and compare the energy values from B-spline approach with low-lying energy levels given by the Sommerfeld formula. The results are presented in the Table I in a.u. Our accuracy of calculation of the energy $10^{-14}$ is the limit of accuracy for the calculation with Fortran double precision type of variables.

Another check of the spline accuracy is given in Table II, where the matrix elements of the operators $r^{m}(m=$ $2,1,0,-1,-2)$ are compared on the $2 s_{1 / 2}$ and $2 p_{1 / 2}$ spline and exact (point-like nucleus) wave functions without weak interaction. The results in Tables I and II show that all the matrix elements $<2 s_{1 / 2}\left|r^{m}\right| 2 s_{1 / 2}>$ and

$<2 p_{1 / 2}\left|r^{m}\right| 2 p_{1 / 2}>$ apart from the case $m=-2$ can be evaluated with the same relative accuracy $10^{-14}-10^{-15}$ as the low-lying energies. Only in case of $m=-2$ the accuracy is of order $10^{-7}-10^{-8}$ because of the singularity of $r^{-2}$ operator.

\section{SCALING AND THE NUMERICAL TESTS}

As the next step we increase the PNC weak interaction by scaling $V_{w}=N_{w} \gamma_{5} W(r)$, where $N_{w}$ is the scaling factor, and investigate the energy spectrum by changing $N_{w}$. Here we use the Fermi distribution for the nuclear charge density.

The scaling problem looks as follows: to find a scaling parameter $N_{w}$ for which 1 ) the contribution of weak interaction to the matrix elements of our interest is larger than numerical inaccuracy, 2) weak interaction is still small enough for using the perturbation theory.

The Table III presents the PNC weak interaction contribution to the energy of the states for the different values of $N_{w}$. In case $N_{w} \neq 0$ the states do not have a certain parity and we use the notations $n \tilde{s}, n \tilde{p}$ formally classifying the different states by their origin. The analysis of the results of calculations shows that the dependence $E=E\left(N_{w}\right)$ is approximately parabolic. One can also obtain this result from the perturbation theory (PT):

$$
\begin{gathered}
|n \tilde{s}\rangle=|n s\rangle+N_{w} \sum_{m p} \frac{\left\langle m p\left|V_{w}(r)\right| n s\right\rangle}{E_{n s}-E_{m p}}|m p\rangle+\frac{1}{2}\left(N_{w}\right)^{2} \sum_{m} \sum_{l} \frac{\left\langle l s\left|V_{w}\right| m p\right\rangle\left\langle m p\left|V_{w}\right| n s\right\rangle}{\left(E_{m p}-E_{l s}\right)\left(E_{n s}-E_{m p}\right)}|l s\rangle \\
\langle n \tilde{s}|\tilde{H}| n \tilde{s}\rangle-E_{n s}=-\left(N_{w}\right)^{2} \sum_{m} \frac{\left|\left\langle m p\left|V_{w}\right| n s\right\rangle\right|^{2}}{E_{n s}-E_{m p}}
\end{gathered}
$$

Here the evident equalities $\left\langle m s\left|V_{w}\right| n s\right\rangle=0$ and $\langle m p|H(r)| n s\rangle=0$ were used. The right-hand side of Eq (11) is also presented in Table III for $N_{w}=1$. The comparison shows that we can obtain the PNC weak interaction contribution to the energy with accuracy $0.03 \%$ if we use scaling parameter $N_{w}=10^{2} \div 10^{3}$. Here by the numerical inaccuracy we undestand the B-spline method inaccuracy of calculation of nonperturbative energy value presented in the last column of Table III. This leads to the poor accuracy of the weak-interaction PNC contributions to the energy, obtained with the low values of scaling parameter $N_{w}=1 \div 10$. For $N_{w}>10^{4}$ higher orders of perturbation theory become to be not negligible.

The second-order PNC weak interaction correction to the energy is of no physical interest and was investigated here only for the demonstration of the possibilities of the scaling procedure. This procedure helps to evaluate the second-order PNC weak interaction correction even though this correction is beyond the level of accuracy of the spline approximation.

We should emphasize that the matrix elements of our interest are the matrix elements with linear dependence on weak interaction. SE and VP corrections to the PNC amplitude are the objects of this type.

For testing our system of PNC B-spline functions we calculated the set of the matrix elements $\left\langle 2 \tilde{s}\left|r^{m}\right| 2 \tilde{s}\right\rangle$ with the first non-zero order of perturbation expansion, linear in $V_{w}$. The following tests were employed:

$$
\begin{aligned}
& \left\langle 2 \tilde{s}\left|r^{m}\right| 2 \tilde{p}\right\rangle=\sum_{n} \frac{\left\langle 2 \tilde{s}\left|r^{m / 2}\right| n \tilde{s}\right\rangle\left\langle n \tilde{s}\left|r^{m / 2}\right| 2 \tilde{p}\right\rangle}{E_{2 \tilde{p}}-E_{n \tilde{s}}}+\sum_{n} \frac{\left\langle 2 \tilde{p}\left|r^{m / 2}\right| n \tilde{p}\right\rangle\left\langle n \tilde{s}\left|r^{m / 2}\right| 2 \tilde{p}\right\rangle}{E_{2 \tilde{p}}-E_{n \tilde{p}}} \\
& \left\langle 2 \tilde{s}\left|r^{m}\right| 2 \tilde{p}\right\rangle=\sum_{n} \frac{\left\langle 2 s\left|r^{m}\right| n s\right\rangle\left\langle n s\left|V_{w}\right| 2 p\right\rangle}{E_{2 p}-E_{n s}}+\sum_{n} \frac{\left\langle 2 s\left|V_{w}\right| n p\right\rangle\left\langle n p\left|r^{m}\right| 2 p\right\rangle}{E_{2 s}-E_{n p}}
\end{aligned}
$$


With these tests we check the completeness of the PNC B-spline spectrum (Eq (12)) and the absence of the higher order PNC contributions after the introduction of the scaling parameter $N_{w}$ i.e. the possibility of rescaling (Eq (13)). The results are given in Table IV. The behaviour of the matrix elements with $m=2,1$ is similar. The most suitable scaling parameters for the calculation of these matrix elements are $N_{w}=1$ (the absence of the scaling) with relative accuracy $1 \cdot 10^{-8}$ and $N_{w}=10$ with relative accuracy $5 \cdot 10^{-8}$. The test of completeness of the spectrum for these operators gives the relative accuracy $1 \cdot 10^{-12}$. The most interesting matrix elements are the ones with $m=-1,-2$ because of their singularity. For $N_{w}=1$ the relative inaccuracy for the calculation of both matrix elements is about $1 \cdot 10^{-6}$, for $N_{w}=10$ it is $1 \cdot 10^{-7}$. The checking of completeness of the basis for this operators give the relative accuracy $1 \cdot 10^{-7}$ in the worst case. Summarizing these tests we conclude that the best scaling parameter for the calculation of matrix element with linear dependence on weak interaction is $N_{w}=10$. The accuracy of this calculation is about $1 \cdot 10^{-7}$.

Finally, we want to illustrate our method by calculation of VP corrections in the Uehling approximation to the matrix element of PNC weak interaction on $\left|2 s_{1 / 2}\right\rangle$ and $\left|2 p_{1 / 2}\right\rangle$ functions. Such electroweak radiative corrections for the HCI were first obtained in [18] by solving Dirac equation with the Uehling potential

$$
V_{u}=\frac{2 \alpha Z}{3 \pi r} \int_{1}^{\infty} e^{-2 r y / \alpha}\left(1+\frac{1}{2 y^{2}}\right) \frac{\sqrt{y^{2}-1}}{y^{2}} d y .
$$

The scheme of calculation of these corrections via PT looks as follows

$$
\begin{gathered}
\left\langle 2 s^{\prime}\left|V_{w}\right| 2 p^{\prime}\right\rangle-\left\langle 2 s\left|V_{w}\right| 2 p\right\rangle=\sum_{n \neq 2} \frac{\left\langle 2 s\left|V_{u}\right| n s\right\rangle\left\langle n s\left|V_{w}\right| 2 p\right\rangle}{E_{2 s}-E_{n s}}+\sum_{n \neq 2} \frac{\left\langle 2 s\left|V_{w}\right| n p\right\rangle\left\langle n p\left|V_{u}\right| 2 p\right\rangle}{E_{2 p}-E_{n p}}+ \\
+\sum_{n \neq 2} \sum_{l \neq 2}\left(\frac{\left\langle 2 s\left|V_{u}\right| n s\right\rangle\left\langle n s\left|V_{w}\right| l p\right\rangle\left\langle l p\left|V_{u}\right| 2 p\right\rangle}{\left(E_{2 s}-E_{n s}\right)\left(E_{2 p}-E_{l p}\right)}+\frac{\left\langle 2 s\left|V_{u}\right| l s\right\rangle\left\langle l s\left|V_{u}\right| n s\right\rangle\left\langle n s\left|V_{w}\right| 2 p\right\rangle}{\left(E_{2 s}-E_{n s}\right)\left(E_{2 s}-E_{l s}\right)}+\frac{\left\langle 2 s\left|V_{w}\right| n p\right\rangle\left\langle n p\left|V_{u}\right| l p\right\rangle\left\langle l p\left|V_{w}\right| 2 p\right\rangle}{\left(E_{2 p}-E_{n p}\right)\left(E_{2 p}-E_{l p}\right)}\right)- \\
-\sum_{n \neq 2} \frac{\left\langle 2 s\left|V_{w}\right| n p\right\rangle\left\langle n p\left|V_{u}\right| 2 p\right\rangle\left\langle 2 p\left|V_{u}\right| 2 p\right\rangle}{\left(E_{2 p}-E_{n p}\right)^{2}}-\sum_{n \neq 2} \frac{\left\langle 2 s\left|V_{u}\right| 2 s\right\rangle\left\langle 2 s\left|V_{u}\right| n s\right\rangle\left\langle n s\left|V_{w}\right| 2 p\right\rangle}{\left(E_{2 s}-E_{n s}\right)^{2}}- \\
-\sum_{n \neq 2} \frac{\left\langle 2 s\left|V_{w}\right| 2 p\right\rangle}{2}\left[\frac{\left|\left\langle 2 s\left|V_{u}\right| n s\right\rangle\right|^{2}}{\left(E_{2 s}-E_{n s}\right)^{2}}+\frac{\left|\left\langle 2 p\left|V_{u}\right| n p\right\rangle\right|^{2}}{\left(E_{2 p}-E_{n p}\right)^{2}}\right]
\end{gathered}
$$

where the notation $\left|2 s^{\prime}\right\rangle$ and $\left|2 p^{\prime}\right\rangle$ means that this functions were obtained as a solutions of the Dirac equation with the Uehling potential. The right-hand side of Eq (15) presents PT expansion. We include the second order of PT because of its significance. Our results for the matrix elements in the left-hand side of Eq (15) are: $\left\langle 2 s\left|V_{w}\right| 2 p\right\rangle=-4.163986 \cdot 10^{-6}$ $\mathrm{eV}$ and $\left\langle 2 s^{\prime}\left|V_{w}\right| 2 p^{\prime}\right\rangle=-4.219566 \cdot 10^{-6} \mathrm{eV}$. These values are slightly different from the values obtained in [18] because of our use of Fermi charge density distribution and more modern data for the nuclear radius. In total the left-hand side of $\mathrm{Eq}(15)$ is equal to $-5,5580 \cdot 10^{-8} \mathrm{eV}$. The calculation in the first order of PT in the right-hand side of Eq (15) gives $-5.5058 \cdot 10^{-8} \mathrm{eV}$. The evaluation of the second order terms yields the value $-5,2277 \cdot 10^{-10} \mathrm{eV}$ and the sum of the first and second orders terms is $-5.5581 \cdot 10^{-8} \mathrm{eV}$. Thus the relative discrepancy between the left and right-hand sides of $\mathrm{Eq}(15)$ is about $2 \cdot 10^{-5}$. This is the limit of accuracy of the calculation of the difference between two matrix elements $\left\langle 2 s\left|V_{w}\right| 2 p\right\rangle$ and $\left\langle 2 s^{\prime}\left|V_{w}\right| 2 p^{\prime}\right\rangle$ that were calculated with accuracy $10^{-7}$.

For our purposes it is interesting to solve the Dirac equation with the PNC weak interaction and to calculate then the matrix element of the Uehling potential in a direct way and via PT:

$$
\left\langle 2 \tilde{s}\left|V_{u}\right| 2 \tilde{p}\right\rangle=\sum_{n} \frac{\left\langle 2 s\left|V_{u}\right| n s\right\rangle\left\langle n s\left|V_{w}\right| 2 p\right\rangle}{E_{2 p}-E_{n s}}+\sum_{n} \frac{\left\langle 2 s\left|V_{w}\right| n p\right\rangle\left\langle n p\left|V_{u}\right| 2 p\right\rangle}{E_{2 s}-E_{n p}}
$$

The results of comparison of the left- and right-hand sides of Eq (16) are given in Table IV. The agreement is about $1 \cdot 10^{-7}$ for $N_{w}=1 \div 10$.

It may be interesting also to compare the evaluation of the Uehling-PNC matrix element in a way given by Eq (15) and by the application of Eq (16). For this comparison we rearrange the terms in the right-hand side of Eq (16), separating out the large contribution, containing small denominator $\Delta E^{(2)}=E_{2 s}-E_{2 p}$. We remind that in our calculation we do not include the radiative corrections in the Dirac energy values. Therefore $\Delta E^{2} \neq 0$ only due to the use of the non-Coulomb field of the nucleus (Fermi distribution). Using the smallness of the differences $\Delta E^{(n)}=E_{n s}-E_{n p}$ we replace $E_{n s}$ by $E_{n p}$ and vice versa in the right-hand side of Eq (16). Then we arrive at the equality

$$
\left\langle 2 \tilde{s}\left|V_{u}\right| 2 \tilde{p}\right\rangle-\frac{\left[\left\langle 2 p\left|V_{u}\right| 2 p\right\rangle-\left\langle 2 s\left|V_{u}\right| 2 s\right\rangle\right]\left\langle 2 s\left|V_{w}\right| 2 p\right\rangle}{E_{2 s}-E_{2 p}} \simeq \sum_{n \neq 2} \frac{\left\langle 2 s\left|V_{u}\right| n s\right\rangle\left\langle n s\left|V_{w}\right| 2 p\right\rangle}{E_{2 s}-E_{n s}}+\sum_{n \neq 2} \frac{\left\langle 2 s\left|V_{w}\right| n p\right\rangle\left\langle n p\left|V_{u}\right| 2 p\right\rangle}{E_{2 p}-E_{n p}},
$$


the right-hand side of which coincides with the first two terms in the right-hand side of Eq (15). Evaluation of the left-hand side of $\mathrm{Eq}(17)$ gives the value $-5.5057 \cdot 10^{-8} \mathrm{eV}$ in a good agreement with the first-order PT result in Eq (15).

The second order of PT can be obtained by calculation of the following expression

$$
\begin{gathered}
\sum_{n \neq 2} \frac{\left\langle 2 \tilde{s}\left|V_{u}\right| n \tilde{s}\right\rangle\left\langle n \tilde{s}\left|V_{u}\right| 2 \tilde{p}\right\rangle}{E_{2 \tilde{s}}-E_{n \tilde{s}}}+\sum_{n \neq 2} \frac{\left\langle 2 \tilde{s}\left|V_{u}\right| n \tilde{p}\right\rangle\left\langle n \tilde{p}\left|V_{u}\right| 2 \tilde{p}\right\rangle}{E_{2 \tilde{s}}-E_{n \tilde{p}}}- \\
-\frac{\left\langle 2 s\left|V_{w}\right| 2 p\right\rangle}{E_{2 s}-E_{2 p}} \sum_{n \neq 2}\left(\frac{\left\langle 2 p\left|V_{u}\right| n p\right\rangle\left\langle n p\left|V_{u}\right| 2 p\right\rangle}{E_{2 p}-E_{n p}}-\frac{\left\langle 2 s\left|V_{u}\right| n s\right\rangle\left\langle n s\left|V_{u}\right| 2 s\right\rangle}{E_{2 s}-E_{n s}}\right) \\
-\sum_{n \neq 2} \frac{\left\langle 2 s\left|V_{w}\right| 2 p\right\rangle}{2}\left[\frac{\left|\left\langle 2 s\left|V_{u}\right| n s\right\rangle\right|^{2}}{\left(E_{2 s}-E_{n s}\right)^{2}}+\frac{\left|\left\langle 2 p\left|V_{u}\right| n p\right\rangle\right|^{2}}{\left(E_{2 p}-E_{n p}\right)^{2}}\right]
\end{gathered}
$$

For this expression we obtain the value $-5,2239 \cdot 10^{-10} \mathrm{eV}$. Then the sum of the first and second orders terms is $-5.5579 \cdot 10^{-8} \mathrm{eV}$. Then, the relative accuracy of calculation of the sum of the first and second order terms is the same as in the first method: $2 \cdot 10^{-5}$. Here we also lose two orders in accuracy due to subtraction of the leading terms in Eq (17). In any case we can state emphasize that the accuracy of calculation of any PNC matrix elements is of order

$10^{-7}$. Of course, one can lose the accuracy of calculation because of subtraction of two large values. The question of the accuracy of calculation of some composite expression should be solved separately in each case. As we understand, the calculation of the radiative corrections to the emission PNC amplitude should not contain any subtractions.

Concluding, we can say that the method developed here presents a powerful tool for the calculation of any corrections to the PNC matrix elements in atoms and ions. The recommended scaling parameter is $N_{w}=1$ or $N_{w}=10$ and the relative accuracy for the calculation of PNC matrix elements achieved in all cases is not less than $10^{-7}$. In particular, this approach can be applied to the calculation of the radiative corrections to the emission PNC amplitude with high accuracy without any approximations ( $\alpha Z$ expansion, Uehling potential approximation and other). Including in our scheme the Dirac-Hartree-Fock potential we can calculate these radiative corrections also for neutral atoms, e.g. for $C s$. It will help to solve finally the problem of the possible deviation of the measured weak charge value $Q_{w}$ from that predicted by the Standard Model.

\section{ACKNOWLEDGMENTS}

The authors thank I.Goidenko and O.Yu.Andreev for the help with the B-spline codes. They are also grateful to G.Soff for drawing their attention to the paper [28]. The financial support by the RFBR grant 02-02-16758 and by Minobrazovanje grant E02-3.1-7 is acknowledged.

[1] C.S. Wood et al., Science 275, 1759 (1997)

[2] S.C. Bennett and C.E. Wieman, Phys.Rev.Lett. 82, 2484 (1999)

[3] A. Derevianko, Phy.Rev.Lett. 85, 1618 (2000)

[4] V.A. Dzuba, C. Harabati, W.R. Johnson and M.S. Safronova, Phys.Rev. A 63, 044103 (2001)

[5] M.G. Kozlov, S.G. Porsev and I.I. Tupitsyn, Phys.Rev.Lett. 86, 3260 (2001)

[6] A. Derevianko, Phys.Rev. A 65, 012106 (2001)

[7] A.A. Vasilyev, I.M. Savukov, M.S. Safronova and H.G. Berry, Phys.Rev. A 66, 020101 (2002)

[8] W.R. Johnson, I. Bednyakov and G. Soff, Phys.Rev.Lett. 17, 233001 (2001)

[9] A.I. Milstein and O.P. Sushkov, Phys.Rev A 66, 022108 (2002)

[10] A.I. Milstein, O.P. Sushkov and I.S. Terekhov Phys.Rev.Lett. 89, 28003 (2002)

[11] M.Yu. Kuchiev, V.V. Flambaum Phys.Rev.Lett. 89, 283002 (2002)

[12] M.Yu. Kuchiev, V.V. Flambaum J.Phys. B 36, R191-R222 (2003)

[13] V.G. Gorshkov and L.N. Labzowsky, Pis'ma Zh.Eksp.Teor.Fiz. 19, 768 (1974) [JETP Lett. 19, 394 (1974)]

[14] A. Schäfer, G. Soff, P. Indelicato, B. Müller and W. Greiner, Phys.Rev. A 40, 7362 (1989)

[15] G. von Oppen, Z.Phys. D 21, 181 (1991)

[16] V.V. Karasiev, L.N. Labzowsky and A.V. Nefiodov, Phys.Lett. A 172, 62 (1992)

[17] R.W. Dunford, Phys.Rev. A 54, 3820 (1996) 
[18] I. Bednyakov, L. Labzowsky, G. Plunien, G. Soff and V. Karasiev, Phys.Rev. A 61, 012103 (1999)

[19] J. Sapirstein, K. Pachucki, A. Veitia and K.T. Cheng, Phys.Rev. A 67, 052110 (2003)

[20] L.N. Labzowsky, A. Prosorov, A.V. Shonin, I. Bednyakov, G. Plunien and G. Soff, Annals of Physics 302, 22 (2002)

[21] P.J. Mohr, G. Plunien and G. Soff, Phys.Rep. 293, 229 (1998)

[22] S.A. Blundell and N.J. Snyderman, Phys.Rev. A 44, R1427 (1991)

[23] A. Mitrushenkov, L. Labzowsky, I. Lindgren, H. Persson and S. Salomonson, Phys.Lett. A 200, 51 (1995)

[24] W.R. Johnson, S.A. Blundell and J. Sapirstein, Phys.Rev. A 37, 307 (1988)

[25] C. Froese-Fischer and F.A. Parpia, Phys.Lett. A 179, 198 (1993)

[26] I.B. Khriplovich, Parity Nonconservation in Atomic Phenomena, Gordon and Breach, New York, 1991

[27] D.E. Groom at al., Euro.Phys.J. C 15, 1 (2000)

[28] A. Schäfer, B. Müller and W. Greiner, Zeit. für Phys. A 322, 539 (1985)

[29] C.W.A. Fletcher, Computational Galerkin Methods, Springer, New York, 1984

[30] A. Chodos, R.L. Jaffe, K. Johnson, C.B. Thorn and V.W. Weisskopf, Phys.Rev. D 9, 3471 (1974)

[31] V.A. Yerokhin, A.N. Artemyev, T. Beier, G. Plunien, V.M. Shabaev and G. Soff, Phys. Rev. A 60, 3522 (1999)

TABLE I. Comparison of the Sommerfeld and spline spectra in absence of $V_{w}$

\begin{tabular}{|c|c|c|c|}
\hline State & Energy(B-spl.appr.) & Energy(Sommerfeld) & Relative inaccuracy \\
\hline $1 s_{1 / 2}$ & -4861.197895993788 & -4861.197895993730 & $1.2 \mathrm{E}-14$ \\
\hline $2 s_{1 / 2}$ & -1257.395849439810 & -1257.395849439807 & $2.4 \mathrm{E}-15$ \\
\hline $2 p_{1 / 2}$ & -1257.395849439792 & -1257.395849439807 & $-1.2 \mathrm{E}-14$ \\
\hline $2 p_{3 / 2}$ & -1089.611415894742 & -1089.611415894764 & $-2.0 \mathrm{E}-14$ \\
\hline $3 s_{1 / 2}$ & -539.0933280909740 & -539.0933280909703 & $6.9 \mathrm{E}-15$ \\
\hline $3 p_{1 / 2}$ & -539.0933280909630 & -539.0933280909703 & $-1.4 \mathrm{E}-14$ \\
\hline $3 p_{3 / 2}$ & -489.0370846743426 & -489.0370846743463 & $-7.6 \mathrm{E}-15$ \\
\hline $3 d_{3 / 2}$ & -489.0370846743426 & -489.0370846743463 & $-7.6 \mathrm{E}-15$ \\
\hline $3 d_{5 / 2}$ & -476.2615942332995 & -476.2615942332814 & $3.8 \mathrm{E}-14$ \\
\hline $4 s_{1 / 2}$ & -295.2578381192325 & -295.2578381192252 & $2.5 \mathrm{E}-14$ \\
\hline $4 p_{1 / 2}$ & -295.2578381192179 & -295.2578381192252 & $-2.5 \mathrm{E}-14$ \\
\hline $4 p_{3 / 2}$ & -274.4077572604947 & -274.4077572604874 & $2.7 \mathrm{E}-14$ \\
\hline $4 d_{3 / 2}$ & -274.4077572604765 & -274.4077572604874 & $-4.0 \mathrm{E}-14$ \\
\hline $4 d_{5 / 2}$ & -268.9658771399190 & -268.9658771399118 & $2.7 \mathrm{E}-14$ \\
\hline $4 f_{5 / 2}$ & -268.9658771399227 & -268.9658771399118 & $4.1 \mathrm{E}-14$ \\
\hline $4 f_{7 / 2}$ & -266.3894469008119 & -266.3894469008155 & $-1.4 \mathrm{E}-14$ \\
\hline $5 s_{1 / 2}$ & -185.4851885786884 & -185.4851885786193 & $3.7 \mathrm{E}-13$ \\
\hline $5 p_{1 / 2}$ & -185.4851885785974 & -185.4851885786193 & $-1.2 \mathrm{E}-13$ \\
\hline $5 p_{3 / 2}$ & -174.9446126829571 & -174.9446126830371 & $-4.6 \mathrm{E}-13$ \\
\hline $5 d_{3 / 2}$ & -174.9446126830371 & -174.9446126830371 & $1.0 \mathrm{E}-15$ \\
\hline $5 d_{5 / 2}$ & -172.1552518809149 & -172.1552518811695 & $-1.5 \mathrm{E}-12$ \\
\hline $5 f_{5 / 2}$ & -172.1552518811732 & -172.1552518811695 & $2.2 \mathrm{E}-14$ \\
\hline $5 f_{7 / 2}$ & -170.8289368147998 & -170.8289368144324 & $2.2 \mathrm{E}-12$ \\
\hline $5 g_{7 / 2}$ & -170.8289368144287 & -170.8289368144324 & $-2.2 \mathrm{E}-14$ \\
\hline $5 g_{9 / 2}$ & -170.0499341722752 & -170.0499341722061 & $4.1 \mathrm{E}-13$ \\
\hline
\end{tabular}


TABLE II. Comparison of the exact (point-like nucleus) and spline values for the matrix elements $<2 s_{1 / 2}\left|r^{m}\right| 2 s_{1 / 2}>$, $<2 p_{1 / 2}\left|r^{m}\right| 2 p_{1 / 2}>$, in absence of $V_{w}$.

\begin{tabular}{rccc}
\hline \hline \multicolumn{4}{c}{ Matrix element $<2 s\left|r^{m}\right| 2 s>$ in a.u. } \\
\hline $\mathrm{m}$ & Spline functions & Exact functions & Relative inaccuracy \\
\hline 2 & $3.428467651648418 \mathrm{E}-3$ & $3.428467651648391 \mathrm{E}-3$ & $-6.4 \mathrm{E}-15$ \\
1 & $5.333841373470161 \mathrm{E}-2$ & $5.333841373470151 \mathrm{E}-2$ & $-2.6 \mathrm{E}-15$ \\
0 & 1.00000000000000 & 1.00000000000000 & $1.0 \mathrm{E}-16$ \\
-1 & 33.2605595132210 & 33.2605595132212 & $1.2 \mathrm{E}-14$ \\
-2 & 8415.21963758472 & 8415.21963813828 & $6.6 \mathrm{E}-11$ \\
\hline \multicolumn{2}{c}{ Matrix element $<2 p\left|r^{m}\right| 2 p>$ in a.u. } \\
\hline $\mathrm{m}$ & Spline functions & Exact functions & Relative inaccuracy \\
\hline 2 & $2.276847838830044 \mathrm{E}-3$ & $2.276847838830025 \mathrm{E}-3$ & $-9.2 \mathrm{E}-15$ \\
1 & $4.246884851731023 \mathrm{E}-2$ & $4.246884851731020 \mathrm{E}-2$ & $7.1 \mathrm{E}-16$ \\
0 & 1.00000000000000 & 1.00000000000000 & $1.0 \mathrm{E}-16$ \\
-1 & 33.2605595132206 & 33.2605595132212 & $1.2 \mathrm{E}-14$ \\
-2 & 2542.94240478497 & 2542.94246617904 & $2.4 \mathrm{E}-08$ \\
\hline \hline
\end{tabular}

TABLE III. Relative contribution of PNC weak interaction to the energies for the different values of $N_{w}$ (the left-hand side of Eq (11) divided by nonperturbative energy value) and the relative contribution of weak interaction to the energies calculated by perturbation theory (the right-hand side of Eq (11) divided by nonperturbative energy value). The last column shows the relative inaccuracy of spline method from Table I.

\begin{tabular}{|c|c|c|c|c|c|c|c|}
\hline$N_{w}$ & 1 & 10 & $10^{2}$ & $10^{3}$ & $10^{4}$ & PT value & $\overline{\delta_{s p l}}$ \\
\hline $1 \tilde{s}_{1 / 2}$ & $-2.65322 \mathrm{E}-13$ & $-2.94537 \mathrm{E}-11$ & $-2.95031 \mathrm{E}-09$ & $-2.95046 \mathrm{E}-07$ & $-2.96521 \mathrm{E}-05$ & $-2.95031 \mathrm{E}-13$ & $2.4 \mathrm{e}-15$ \\
\hline $2 \tilde{p}_{1 / 2}$ & $-7.13101 \mathrm{E}-13$ & $-3.13915 \mathrm{E}-11$ & $-3.14292 \mathrm{E}-09$ & $-3.14285 \mathrm{E}-07$ & $-3.13759 \mathrm{E}-05$ & $-3.14291 \mathrm{E}-13$ & $-1.2 \mathrm{E}-14$ \\
\hline $2 \tilde{s}_{1 / 2}$ & $6.36938 \mathrm{E}-14$ & $6.64008 \mathrm{E}-12$ & $6.57424 \mathrm{E}-10$ & 6.57067E-08 & 6.39177E-06 & $6.57255 \mathrm{E}-14$ & $-2.0 \mathrm{E}-14$ \\
\hline
\end{tabular}

TABLE IV. The matrix elements $<2 \tilde{s}_{1 / 2}\left|r^{m}\right| 2 \tilde{p}_{1 / 2}>(m=2,1,-1,-2)$ calculated by different methods with the different values of the scaling parameter $N_{w}=10^{r}$. The first line in the every box for a certain $m$ and $r$ values corresponds to the direct evaluation of the matrix element with B-spline solution of the Dirac equation with PNC weak interaction. The second line corresponds to the use of the formula Eq (12) in the text. The PT line corresponds to the perturbation theory evaluation via the formula Eq (13) with $N_{w}=1$. (in a.u.) The last column presents the results of evaluation of the Uehling matrix element with the same methods.

\begin{tabular}{|c|c|c|c|c|c|}
\hline \multirow[t]{2}{*}{$\mathrm{r}$} & \multicolumn{4}{|c|}{$\mathrm{m}$} & \multirow[t]{2}{*}{$\left\langle 2 \tilde{s}\left|V_{u}\right| 2 \tilde{p}\right\rangle \mathrm{eV}$} \\
\hline & 2 & 1 & -1 & -2 & \\
\hline \multirow[t]{2}{*}{0} & $-1.971129293262 \mathrm{E}-8$ & $-1.858655956533 \mathrm{E}-7$ & $-1.914713446350 \mathrm{E}-7$ & $-8.871229471602 \mathrm{E}-2$ & $1.75928086582129 \mathrm{E}-6$ \\
\hline & $-1.971129293278 \mathrm{E}-8$ & $-1.858655956541 \mathrm{E}-7$ & $-1.914713461356 \mathrm{E}-7$ & $-8.871223340185 \mathrm{E}-2$ & $1.75928086582128 \mathrm{E}-6$ \\
\hline 1 & $-1.971129190573 \mathrm{E}-7$ & $-1.858655859884 \mathrm{E}-6$ & $-1.914710899371 \mathrm{E}-6$ & $-8.871236585343 \mathrm{E}-1$ & $1.75928077138618 \mathrm{E}-5$ \\
\hline \multirow[t]{2}{*}{2} & $-1.971122272187 \mathrm{E}-6$ & $-1.858649339848 \mathrm{E}-5$ & $-1.914707122846 \mathrm{E}-5$ & $-8.87120565454 \mathrm{E}+0$ & $1.75927469110662 \mathrm{E}-4$ \\
\hline & $-1.971122272187 \mathrm{E}-6$ & $-1.858649339848 \mathrm{E}-5$ & $-1.914707122268 \mathrm{E}-5$ & $-8.87120593112 \mathrm{E}+0$ & $1.75927469110664 \mathrm{E}-4$ \\
\hline 3 & $-1.970430890846 \mathrm{E}-5$ & $-1.857997762637 \mathrm{E}-4$ & $-1.914283967449 \mathrm{E}-4$ & $-8.86823302428 \mathrm{E}+1$ & $1.75866695639620 \mathrm{E}-3$ \\
\hline \multirow[t]{2}{*}{4} & $-1.904352098651 \mathrm{E}-4$ & $-1.795723793234 \mathrm{E}-3$ & $-1.874284426839 \mathrm{E}-3$ & $-8.58437274571 \mathrm{E}+2$ & $1.70059752407138 \mathrm{E}-2$ \\
\hline & $-1.904352098651 \mathrm{E}-4$ & $-1.795723793234 \mathrm{E}-3$ & $-1.874284426869 \mathrm{E}-3$ & $-8.58437274521 \mathrm{E}+2$ & $1.70059752407139 \mathrm{E}-2$ \\
\hline $\mathrm{PT}$ & $-1.971129278718 \mathrm{E}-8$ & $-1.858655943008 \mathrm{E}-7$ & $-1.914711189050 \mathrm{E}-7$ & $-8.871235465273 \mathrm{E}-2$ & $1.75928083527704 \mathrm{E}-6$ \\
\hline
\end{tabular}

